\begin{tabular}{|c|l|}
\hline Title & ZNRF1 interacts with tubulin and regulates cell morphogenesis \\
\hline Author(s) & Yoshida, Koichi; W atanabe, Masashi; Hatakeyama, Shigetsugu \\
\hline Citation & $\begin{array}{l}\text { Biochemical and Biophysical Research Communications, 389/3), 506-511 } \\
\text { https://doi.org/10.1016/.bbrc.2009.09.011 }\end{array}$ \\
\hline Issue Date & 2009-11-20 \\
\hline Doc URL & http://hdl.handle.net/2115/39666 \\
\hline Type & article(author version) \\
\hline File Information & BBRC389-3_p506-511.pdf \\
\hline
\end{tabular}

Instructions for use 


\section{ZNRF1 interacts with tubulin and regulates cell morphogenesis}

Koichi Yoshida, Masashi Watanabe, Shigetsugu Hatakeyama *

Department of Biochemistry, Hokkaido University Graduate School of Medicine, Sapporo, Hokkaido 060-8638, Japan.

* Corresponding authors. Shigetsugu Hatakeyama, Department of Biochemistry, Hokkaido University Graduate School of Medicine, N15, W7, Kita-ku, Sapporo, Hokkaido 060-8638, Japan. Phone: +81 11706 5899; Fax: +81 11706 5169; E-mail address: hatas@med.hokudai.ac.jp.

Key words: ZNRF1; tubulin; ubiquitin; RING-finger; zinc-finger 


\begin{abstract}
The ubiquitin-proteasome system has been implicated in neuronal degeneration and regeneration. We demonstrated that overexpression of ZNRF1, which has been identified as a crucial molecule in nerve regeneration, causes morphological changes such as neurite-like elongation. Molecular dissections showed that both the RING finger domain and zinc finger domain are required for morphological changes. Furthermore, we identified $\beta$-tubulin type 2 (Tubb2) as a ZNRF1-binding protein by yeast two-hybrid screening. In vivo binding assay showed that ZNRF1 interacts with tubb2 and immunofluorescent staining suggests that ZNRF1 is colocalized with tubb2. These results suggest that ZNRF1 mediates regulation of neuritogenesis via interaction with tubulin.
\end{abstract}




\section{Introduction}

The ubiquitin-mediated proteolytic pathway plays an important role in the elimination of short-lived regulatory proteins, including those that contribute to the cell cycle, cellular signaling, secretion, organelle biogenesis, DNA repair, and morphogenesis [1]. Ubiquitin conjugation is catalyzed by enzymes designated as ubiquitin-activating enzyme (E1), ubiquitin-conjugating enzyme (E2), and ubiquitin ligase (E3) [2]. E3 is thought to be the component of the ubiquitin conjugation system that is most directly responsible for substrate recognition [1]. On the basis of structural similarity, E3 enzymes have been classified into three families: HECT (homologous to E6-AP $\underline{\mathrm{COOH}}$ terminus) family [3], RING finger-containing protein family $[4,5]$ and U-box family [6].

In the nervous system, the ubiquitin-proteasome pathway is thought to play an important role to ensure proper neuronal function including neuritogenesis and neurodegeneration $[7,8]$. Ubiquitin-mediated degradation not only removes misfolded proteins in the subcellular regions of neurons to prevent the development of neurodegenerative diseases but also mediates transduction of intracellular signaling. It has been reported that neurite outgrowth was induced by proteasome inhibitors, suggesting that neurite outgrowth requires an enhanced pool of ubiquitinated proteins or ubiquitin-dependent proteolysis of a negative regulator of neurite outgrowth in vitro [8].

It has been reported that ZNRF1, identified as one of the nerve injury-induced genes, contains a zinc finger and RING finger domain and is predominantly expressed 
in the nervous system [9] and that ZNRF1 is localized in the endosome-lysosome compartment in fibroblasts [10]. ZNRF1 has an E3 ubiquitin ligase activity and the RING finger domain is required for its activity.

In this study, we found that overexpression of ZNRF1 causes morphological changes such as neurite-like elongation. Furthermore, we identified $\beta$-tubulin type 2 (Tubb2) as a ZNRF1-binding protein by yeast two-hybrid screening, and immunofluorescent staining verified that ZNRF1 is colocalized with Tubb2. These findings suggest that ZNRF1 regulates morphological changes of neurons via interaction with tubulin. 


\section{Materials and methods}

Cell culture. HEK293T, COS-7 and HeLa cell lines were cultured under an atmosphere of $5 \% \mathrm{CO}_{2}$ at $37^{\circ} \mathrm{C}$ in DMEM (Sigma) supplemented with $10 \%$ fetal bovine serum (Invitrogen).

Cloning of cDNAs and plasmid construction. Mouse ZNRF1 and Tubb2 cDNAs were amplified from mouse brain cDNA by PCR with KOD-Plus (Toyobo) using the following primers: 5'-AACATGGGGGGCAAGCAGAGC-3' (ZNRF1-sense), 5'-GGGTCAGTCAGCAGGGTGCTC-3' (ZNRF1-antisense), 5'-ACCATGCGCGAGATCGTGCAC-3' 5'-TTCTCAAGCCTCATCTTCACC-3' (Tubb2-antisense). The amplified fragments were subcloned into pBluescript II SK+ (Stratagene). ZNRF1 cDNA tagged with a carboxy-terminal FLAG tag or Myc tag was cloned into pCMV (Sigma) or pcDNA3 (Invitrogen). Tubb2 cDNAs with an HA tag at the amino terminus were cloned into pCGN. Deletion mutants of ZNRF1 corresponding to 1-144 or 138-227 amino acid residues were generated by $\mathrm{PCR}$, and ZNRF1 mutants (C145A and C184A) were generated by using a QuikChange mutagenesis kit (Stratagene).

Transfection, immunoprecipitation and immunoblot analysis. HEK293T cells were transfected by the calcium phosphate method. After $48 \mathrm{~h}$, the cells were lysed in a solution containing $50 \mathrm{mM}$ Tris- $\mathrm{HCl}(\mathrm{pH} 7.4), 150 \mathrm{mM} \mathrm{NaCl}, 1 \%$ Triton-X 100, leupeptin $(10 \mu \mathrm{g} / \mathrm{ml}), 1 \mathrm{mM}$ phenylmethylsulfonyl fluoride, $400 \mu \mathrm{M} \mathrm{Na} \mathrm{VO}_{4}, 400 \mu \mathrm{M}$ EDTA, $10 \mathrm{mM} \mathrm{NaF}$, and $10 \mathrm{mM}$ sodium pyrophosphate. The cell lysates were 
centrifuged at $16,000 \times \mathrm{g}$ for $20 \mathrm{~min}$ at $4^{\circ} \mathrm{C}$, and the resulting supernatant was incubated with antibodies for $2 \mathrm{~h}$ at $4^{\circ} \mathrm{C}$. Protein A-Sepharose (Amersham Biosciences, Piscataway, NJ) that had been equilibrated with the same solution was added to the mixture, which was then rotated for $1 \mathrm{~h}$ at $4^{\circ} \mathrm{C}$. The resin was separated by centrifugation, washed five times with ice-cold lysis buffer, and then boiled in SDS sample buffer. Immunoblot analysis was performed with the primary antibodies, horseradish peroxidase-conjugated antibodies to mouse or rabbit immunoglobulin $\mathrm{G}$ (1:20,000 dilution, Promega Corporation, Madison, WI) and an enhanced chemiluminescence system (ECL, Amersham Pharmacia) [6]. The following antibodies were used: mouse monoclonal anti-Myc antibody (9E10, Roche Molecular Biochemicals), mouse monoclonal anti-HA antibody (HA.11/16B12, Covance Research Products) and rabbit polyclonal anti-FLAG antibody (Sigma).

Immunofluorescent staining. Cells on a glass cover were fixed for $20 \mathrm{~min}$ at room temperature with 4\% formaldehyde in PBS and then incubated for $1 \mathrm{~h}$ at room temperature with primary antibodies in PBS containing $0.1 \%$ bovine serum albumin and $0.1 \%$ saponin. They were then incubated with Alexa488-labeled goat polyclonal antibodies to mouse immunoglobulin or Alexa546-labeled goat polyclonal antibodies to rabbit immunoglobulin (Molecular Probes) at a dilution of 1:2,000. The cells were covered with a drop of GEL/MOUNT (Biomeda) and then photographed with a CCD camera (DP71, Olympus) attached to an Olympus BX51 microscope [6]. To detect endogenous tubulin, the cells were permeabilized with $100 \%$ acetone at $-20^{\circ} \mathrm{C}$ for $4 \mathrm{~min}$ and then stained with anti- $\beta$-tubulin antibodies (Cell Signaling Technology) [11]. 
Yeast two-hybrid screening. Complementary DNA encoding amino acids 1-144 of mouse ZNRF1 was fused in-frame to the nucleotide sequence for the LexA domain (BD) in the yeast two-hybrid vector pBTM116, and yeast strain L40 (Invitrogen) stably expressing the corresponding pBTM116 vector was transfected with a mouse brain Matchmaker cDNA library (Clontech) by the lithium acetate method.

Semiquantitative $R T-P C R$. The expression levels of ZNRF1 were evaluated by semiquantitative reverse transcription-polymerase chain reaction (RT-PCR). In brief, total RNA was extracted from cultured cells using ISOGEN reagent (Nippon gene) according to the manufacturer's instructions and was used to generate cDNA by reverse transcription with ReverTra Ace (Toyobo). The resulting cDNA was amplified by PCR with KOD-plus (Toyobo) using the following primers: 5'-AGCATGGGGGGCAAGCAGAGC-3' (human and monkey ZNRF1-sense), 5'-AGGTCAGTCCGCAGGGTGTTC-3' (human and monkey ZNRF1-antisense), 5'-ACCACAGTCCATGCCATCAC-3' ' (GAPDH-forward) and 5'-TCCACCACCCTGTTGCT-GTA-3' (GAPDH-reverse). The PCR mixtures were initially denatured at $94^{\circ} \mathrm{C}$ for $2 \mathrm{~min}$, followed by denaturation for $15 \mathrm{~s}$ at $94^{\circ} \mathrm{C}$, primer annealing for $30 \mathrm{~s}$ at $64^{\circ} \mathrm{C}$, and extension for $1 \mathrm{~min}$ at $68^{\circ} \mathrm{C}$ for 30 cycles (for ZNRF1) or 22 cycles (for GAPDH).

Statistical analysis. We used the unpaired Student's $t$ test to determine the statistical significance of experimental data. 


\section{Results}

Overexpression of ZNRF1 causes morphological changes

It has been reported that ZNRF1 proteins are located in the endosome-lysosome compartment in fibroblasts [10]. We examined the subcellular localization of ZNRF1 proteins in another cell line by immunofluorescent staining. We transfected an expression vector encoding carboxy-terminal Myc-tagged ZNRF1 into COS-7 cells. Immunofluorescent staining showed that Myc-tagged ZNRF1 is diffusely localized in the cytosol. More amazingly, overexpression of ZNRF1 caused changes in cell shape (neurite-like elongation). We verified morphological changes in HEK293T and HeLa cells by overexpression of Myc-tagged ZNRF1 (Fig.1A). We found that the shape of COS-7 cells was dramatically changed compared to that of HEK293T or HeLa cells. To clarify differences in the effects among these cell lines, we measured the expression levels of ZNRF1 mRNA in these cell lines. Semi-quantitative analysis using RT-PCR showed that ZNRF1 mRNA was expressed in these cell lines and that ZNRF-1 is highly expressed in COS-7 cells compared with the expression level of HEK293T or HeLa cells (Fig. 1B and C). These findings suggest that a high level of ZNRF1 expression is related to cell morphological changes.

RING finger domain is required for morphological changes 
ZNRF1 has a putative myristoylation sequence at the amino terminus and a zinc finger domain and a RING finger domain at the carboxy-terminal half. It has been reported that ZNRF1 has an E3 ubiquitin ligase activity and requires the RING finger domain for its activity. To examine which region is required for the morphological change, we constructed several deletion mutants or point mutants of ZNRF1 (Fig. 2A). A previous study indicated that ZNRF1(C184A), in which Cys184 in a RING finger domain is substituted for Ala, has no E3 ubiquitin ligase and that ZNRF1(C145A), in which Cys145 in a zinc finger domain is substituted to Ala, likely causes its conformation. It has been reported that the amino-terminal domain with myristoylation is required for localization to the endosome-lysosome [10]. To inhibit amino-terminal myristoylation, ZNRF1 tagged with an N-terminal Myc-tag was also generated (Fig. 2A). Immunoblot analysis with anti-Myc antibody showed that wild-type and mutants of ZNRF1 proteins were expressed in transfected cells (Fig. 2B). We next transfected expression vectors encoding GFP, ZNRF1(FL) or mutants of ZNRF1 into COS-7 cells (Fig. 2C) and then counted the number of cells in which neurite-like projection is larger than its cell body (Fig. 2D). ZNRF1( $\triangle$ Zinc/RING) and ZNRF1(C184A) sometimes caused cause cell morphological change, whereas ZNRF(C145A) or ZNRF1(138-227) had only a partial effect. Amino-terminal Myc-tagged ZNRF1 (Myc-ZNRF1(FL)) showed almost the same effect as that of carboxy-terminal Myc-tagged ZNRF1 (ZNRF1(FL)-Myc). These findings suggest that the RING finger domain of ZNRF1 is required for morphological changes and that the amino-terminal half of ZNRF1 and zinc finger domain are also partially important for this activity. 
ZNRF1 interacts with Tubb2

To clarify the mechanism by which ZNRF1 affects cell morphology, we tried to identify the protein interacting with ZNRF1 by yeast two-hybrid screening. We screened a mouse brain cDNA library using mouse ZNRF1( $\triangle$ Zinc/RING) as a bait. Consequently, fifty-five positive clones were isolated from $2.2 \times 10^{6}$ transformants. One of the positive clones had sequence identities with mouse $\beta$-tubulin type 2 (Tubb2, also known as $\mathrm{M} \beta 2$ ), which is one of the major components of microtubules. We confirmed that ZNRF1 interacts with Tubb2 by using a $\beta$-galactosidase assay (Fig. 3A). Next, we investigated whether ZNRF1 physically interacts with Tubb2 in mammalian cells. We transfected expression vectors encoding Myc-tagged ZNRF1 and/or HA-tagged Tubb2 into HEK293T cells. Cell lysates were subjected to immunoprecipitation with an antibody to Myc and the resulting precipitates were subjected to immunoblot analysis with an antibody to HA. An in vivo binding assay showed that Tubb2 interacts with ZNRF1(FL) and ZNRF1( $\Delta$ Zinc/RING) but not with ZNRF1(138-227). These results indicate that ZNRF1 specifically interacts with Tubb2 via the amino-terminal region (Fig. 3B).

ZNRF1 colocalizes with tubulin

To verify the intracellular localization of ZNRF1 and Tubb2, we performed 
immunofluorescent staining of FLAG-tagged ZNRF1 and HA-tagged Tubb2. Immunofluorescent staining showed that FLAG-tagged ZNRF1 is colocalized with HA-tagged Tubb2 (Fig. 4A). Furthermore, to confirm the interaction with endogenous tubulin, we performed immunofluorescent staining. We transiently transfected cells with expression vectors encoding GFP and carboxy-terminal Myc-tagged ZNRF1 and then stained them with anti-Myc or anti- $\beta$-tubulin antibodies. Immunofluorescent staining showed that Myc-tagged ZNRF1 is localized with endogenous tubulin arranged on microtubules (Fig. 4B). These findings indicate that ZNRF1 interacts with tubulin and is localized with microtubules. 


\section{Discussion}

In this study, we showed that ZNRF1 interacts with Tubb2, one of the members of the $\beta$-tubulin family. $\beta$-tubulin is the major structural component of the microtubule network inside cells. It has been reported that Tubb2 transcripts are abundantly expressed in the adult mouse brain [12]. ZNRF1 is also highly expressed in the central nervous system, particularly in the developing cortical plate in embryos, indicating that ZNRF1 and Tubb2 may play an important role in the nervous system. It has also been reported that the mRNA expression of Tubb2 was elevated within two weeks after crushing the sciatic nerve [13], and ZNRF1 was identified as a gene induced after sciatic nerve injury [9]. These findings suggest that the collaboration of ZNRF1 with Tubb2 regulates axonal guidance or elongation after nerve injury.

We newly found that ZNRF1 induces cell morphological changes and that a RING finger domain is required for this neurite-like elongation. Unfortunately, we could not show ubiquitination of Tubb2 or acceleration of the turnover of Tubb2 by ZNRF1 (data not shown). However, since a RING finger domain of ZNRF1 is important for E3 ubiquitin ligase activity and for morphological changes, we cannot rule out the possibility that proteins other than Tubb2 are substrates for ZNRF1. It has also been reported that a large multidomain E3 ubiquitin ligase $\mathrm{Phr} 1$ is associated with the microtubule cytoskeleton within neurons and selectively localizes to axons but is excluded from growth cones and that motor and sensory neurons from Magellan mice in which the Phrl gene is mutated displays abnormal morphologies. It has been reported 
that the E3 ubiquitin ligase Parkin, the gene of which has several mutations in autosomal-recessive juvenile Parkinsonism (ARJP), binds to tubulin heterodimers and accelerates degradation of $\alpha$ - and $\beta$ - tubulin [14], resulting in possible protection by Parkin of neurons from the toxic accumulation of misfolded tubulin. Taken together, tubulin modification by ubiquitination may be important for neuritogenesis and intracellular quality-control for neurons.

We previously reported that interaction between U-box type ubiquitin ligase E4B and FEZ1 in mammalian cells was enhanced by coexpression of constitutively active PKC $\zeta$ and that E4B mediated the polyubiquitination of FEZ1. Expression of a dominant-negative mutant of $\mathrm{E} 4 \mathrm{~B}$ in rat pheochromocytoma $\mathrm{PC} 12$ cells resulted in inhibition of neurite extension induced either by nerve growth factor or by coexpression of FEZ1 and constitutively active $\mathrm{PKC} \zeta$ [15]. Others have reported that the E3 ubiquitin ligase Smurf1, which regulates cell polarity and protrusive activity via $\mathrm{PKC} \zeta$-dependent recruitment to cellular protrusion sites, enhances neurite outgrowth [16]. Therefore, it may be important to investigate the relationship between $\mathrm{ZNRF} 1$ and $\mathrm{PKC} \zeta$ in order to clarify the mechanism by which morphological change is induced by overexpression of ZNRF1.

In conclusion, ZNRF1 is a novel regulator affecting cell morphology, and results of further studies on ZNRF1 may be useful for revealing neuronal degeneration or regeneration. 


\section{Acknowledgements}

The work was supported in part by a research grant from Grant-in-Aid for Scientific Research on Priority Areas from the Ministry of Education, Culture, Sports, Science and Technology, Mitsubishi Pharma Sources of Funding, the Cell Science Research Foundation, and the Research Foundation Ituu Laboratory (to S. Hatakeyama). 


\section{References}

[1] A. Hershko, A. Ciechanover, The ubiquitin system, Annu Rev Biochem 67 (1998) 425-479.

[2] M. Scheffner, U. Nuber, J.M. Huibregtse, Protein ubiquitination involving an E1-E2-E3 enzyme ubiquitin thioester cascade, Nature 373 (1995) 81-83.

[3] J.M. Huibregtse, M. Scheffner, S. Beaudenon, P.M. Howley, A family of proteins structurally and functionally related to the E6-AP ubiquitin-protein ligase.[erratum appears in Proc Natl Acad Sci U S A. 1995 May 23;92(11):5249; PMID: 7761480], Proc Nat Acad Sci USA 92 (1995) 2563-2567.

[4] C.A. Joazeiro, A.M. Weissman, RING finger proteins: mediators of ubiquitin ligase activity, Cell 102 (2000) 549-552.

[5] K.L. Lorick, J.P. Jensen, S. Fang, A.M. Ong, S. Hatakeyama, A.M. Weissman, RING fingers mediate ubiquitin-conjugating enzyme (E2)-dependent ubiquitination, Proc Nat Acad Sci USA 96 (1999) 11364-11369.

[6] S. Hatakeyama, M. Yada, M. Matsumoto, N. Ishida, K.I. Nakayama, U box proteins as a new family of ubiquitin-protein ligases, J Biol Chem 276 (2001) 33111-33120.

[7] C.E. Oh, R. McMahon, S. Benzer, M.A. Tanouye, bendless, a Drosophila gene affecting neuronal connectivity, encodes a ubiquitin-conjugating enzyme homolog, J Neurosci 14 (1994) 3166-3179.

[8] M. Obin, E. Mesco, X. Gong, A.L. Haas, J. Joseph, A. Taylor, Neurite outgrowth in 
PC12 cells. Distinguishing the roles of ubiquitylation and ubiquitin-dependent proteolysis, J Biol Chem 274 (1999) 11789-11795.

[9] T. Araki, R. Nagarajan, J. Milbrandt, Identification of genes induced in peripheral nerve after injury. Expression profiling and novel gene discovery, J Biol Chem 276 (2001) 34131-34141.

[10] T. Araki, J. Milbrandt, ZNRF proteins constitute a family of presynaptic E3 ubiquitin ligases, J Neurosci 23 (2003) 9385-9394.

[11] A. Blouin, J.A. Blaho, Assessment of the subcellular localization of the herpes simplex virus structural protein VP22 in the absence of other viral gene products, Virus Res 81 (2001) 57-68.

[12] S.A. Lewis, M.G. Lee, N.J. Cowan, Five mouse tubulin isotypes and their regulated expression during development, J Cell Biol 101 (1985) 852-861.

[13] P.N. Hoffman, D.W. Cleveland, Neurofilament and tubulin expression recapitulates the developmental program during axonal regeneration: induction of a specific beta-tubulin isotype, Proc Natl Acad Sci USA 85 (1988) 4530-4533.

[14] Y. Ren, J. Zhao, J. Feng, Parkin binds to alpha/beta tubulin and increases their ubiquitination and degradation, J Neurosci 23 (2003) 3316-3324.

[15] F. Okumura, S. Hatakeyama, M. Matsumoto, T. Kamura, K.I. Nakayama, Functional regulation of FEZ1 by the U-box-type ubiquitin ligase E4B contributes to neuritogenesis, J Biol Chem 279 (2004) 53533-53543.

[16] B. Bryan, Y. Cai, K. Wrighton, G. Wu, X.H. Feng, M. Liu, Ubiquitination of RhoA by Smurf1 promotes neurite outgrowth, FEBS Lett 579 (2005) 1015-1019. 


\section{Figure legends}

Fig. 1. Overexpression of ZNRF1 induces neurite-like morphological change. (A) Immunofluorescent staining of ZNRF1. HEK293T, HeLa and COS-7 cell lines were transfected with expression vectors encoding GFP or Myc-tagged ZNRF1. Cells were fixed and stained with anti-Myc antibodies. Scale bar: $20 \mu \mathrm{m}$. (B) Expression levels of mRNA of ZNRF1 in HEK293T, HeLa and COS-7 cells were used for semiquantitative RT-PCR. GAPDH was used as an internal control. (C) Semiquantitative analysis of ZNRF1 mRNA levels. Intensity of the PCR bands for ZNRF1 in Fig. 1B was normalized by that of the corresponding GAPDH bands.

Fig. 2. RING finger domain is required for cell morphological change. (A) Schematic representation of wild-type and mutants of ZNRF1 is shown. FL: full-length, Myc: Myc-tagged, Zinc: zinc finger domain, RING: RING finger domain. (B) Immunoblot analysis of generated ZNRF1 mutants. HEK293T cells were transiently transfected with wild-type or mutant types of Myc-tagged ZNRF1 and the cell lysates were subjected to immunoblot analysis with anti-Myc antibody. (C) Morphological changes by transfection with ZNRF1 mutants. COS-7 cells were transiently transfected with GFP or ZNRF1 mutants as indicated in Fig. 1A and stained with anti-Myc antibody. Scale bar: $20 \mu \mathrm{m}$. (D) Quantification of sprouting cells by transfection with ZNRF1 mutants. The number of cells in which neurite-like projection was larger than its cell body was counted. The percentage of sprouting cells is shown as mean \pm S.D. of values from three 
independent experiments.

Fig. 3. ZNRF1 interacts with Tubb2. (A) Yeast two-hybrid screening for identifying ZNRF1-interacting proteins. Mouse Tubb2 was identified as a ZNRF1-interacting protein using a mouse brain cDNA library. An empty vector (Mock) was used as a negative control, and CHIP and EKN1 cDNAs were used for positive controls. BD: DNA-binding domain, AD: DNA-activating domain. (B) In vivo binding assay between ZNRF1 and Tubb2. Expression vectors encoding Myc-tagged ZNRF1 mutants indicated in Fig. 2A and HA-tagged Tubb2 were transfected into HEK293T cells. Whole cell lysates (WCL) were immunoprecipitated with anti-Myc antibody and immunoblotted with anti-HA and anti-Myc antibodies.

Fig. 4. Colocalization of ZNRF1 with tubulin. (A) ZNRF1 colocalizes with Tubb2. COS-7 cells were transfected with expression vectors encoding FLAG-tagged ZNRF1 and HA-tagged Tubb2. Cells were stained with anti-FLAG (red) and anti-HA (green) antibodies. Scale bar: $20 \mu \mathrm{m}$. (B) ZNRF1 colocalizes with endogenous $\beta$-tubulin. COS-7 cells were transiently transfected with Myc-tagged ZNRF1. Cells were stained with anti-Myc (red) and anti- $\beta$-tubulin (green) antibodies. Scale bar: $20 \mu \mathrm{m}$. 
Figy象 1

GFP
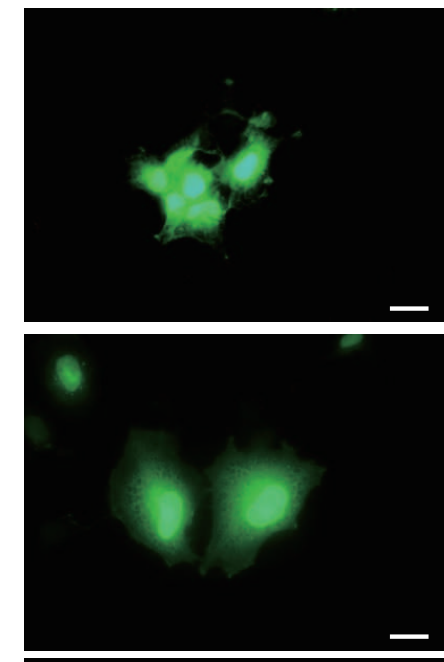

HeLa

cos-7
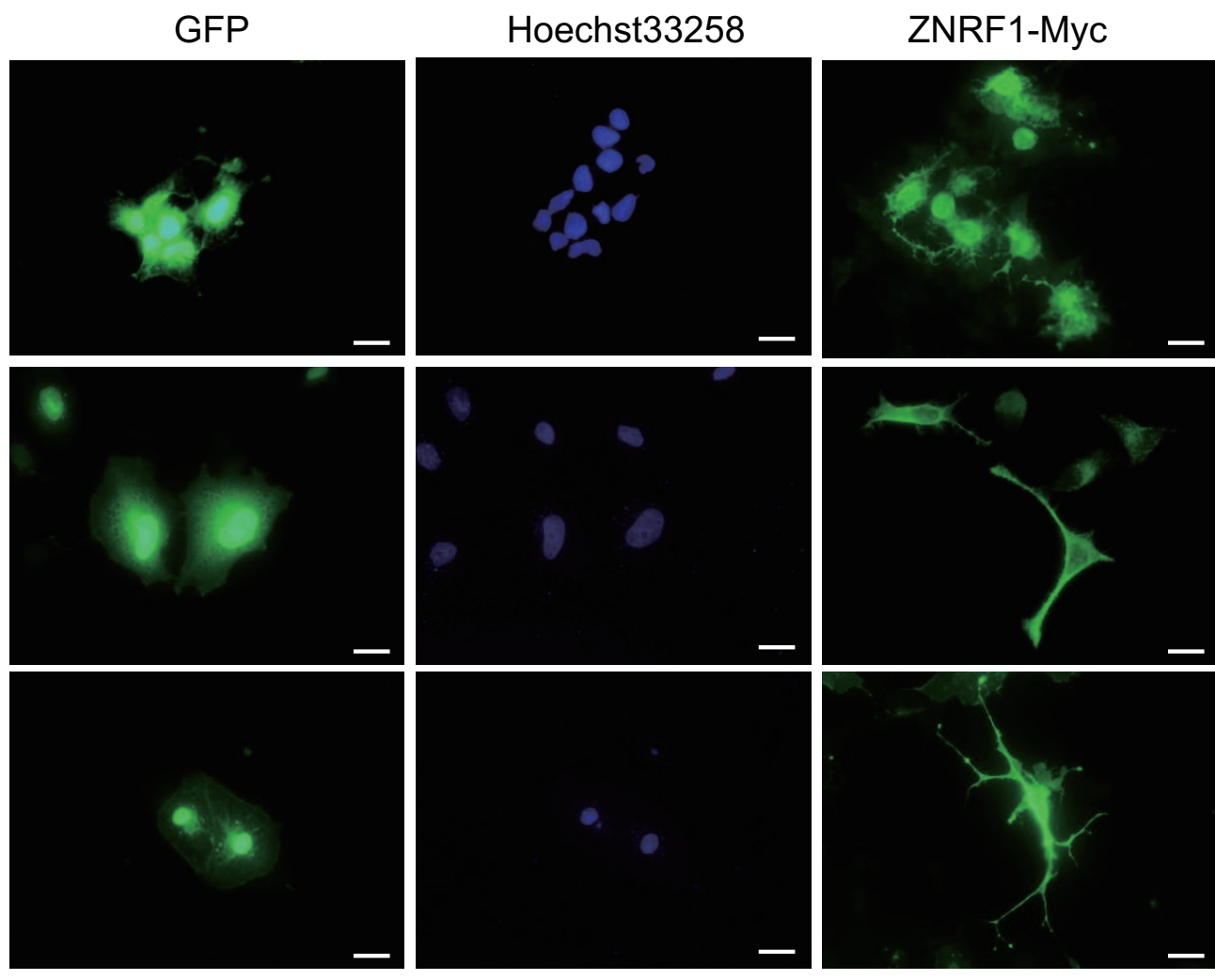

Hoechst33258
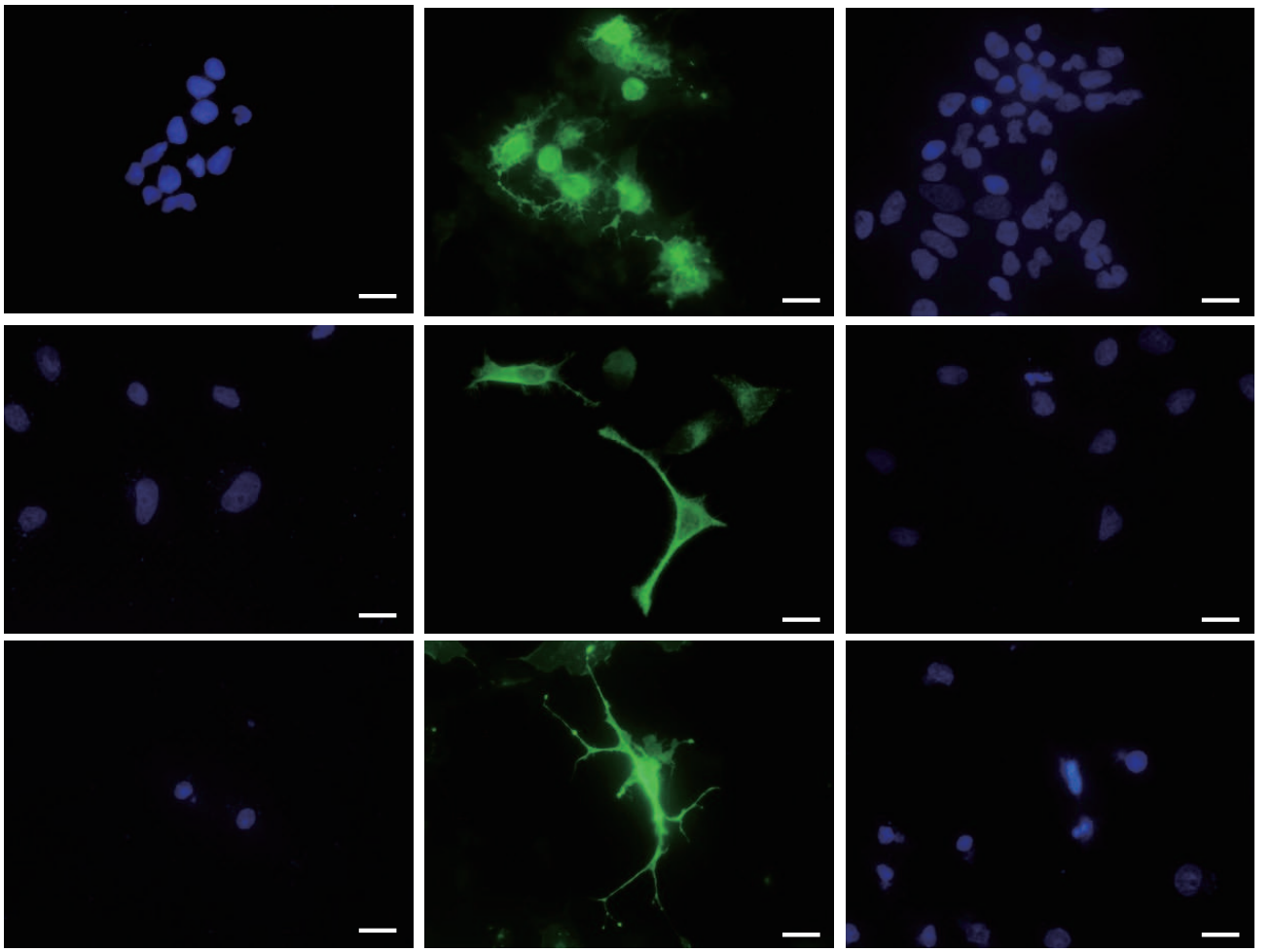

B

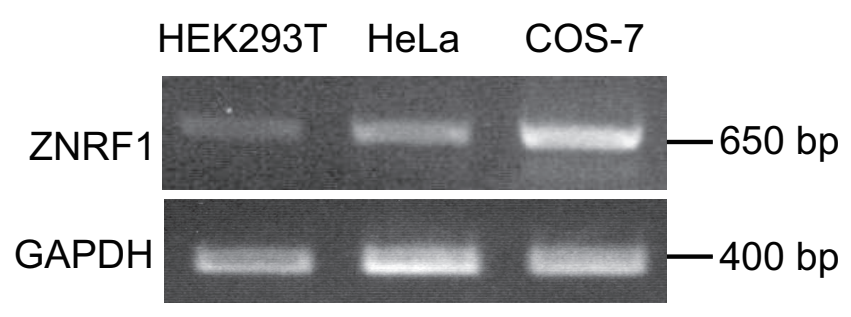

C

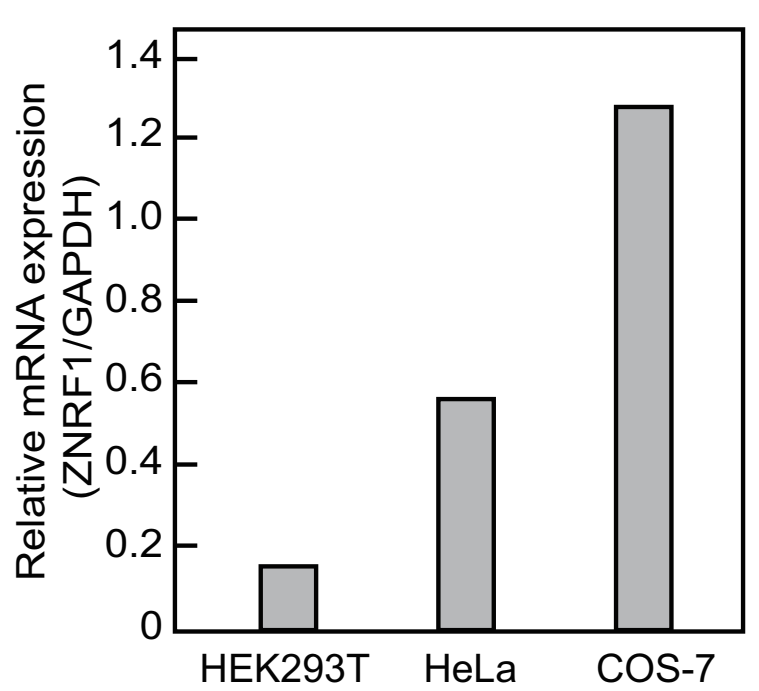




\section{$A^{\text {Agure 2 }}$}

ZNRF1(FL)-Myc

ZNRF1( $\Delta$ Zinc/RING)-Myc $\frac{14}{1}$

ZNRF1(C184A)-Myc
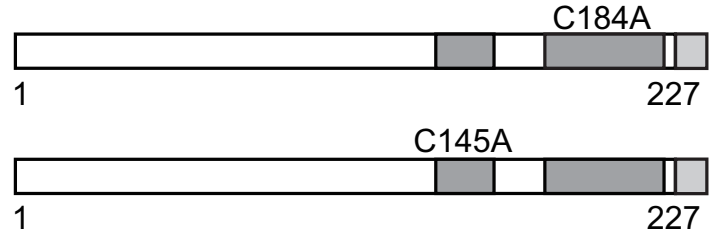

ZNRF1(C145A)-Myc

Myc-ZNRF1(138-227)

Myc-ZNRF1(FL)
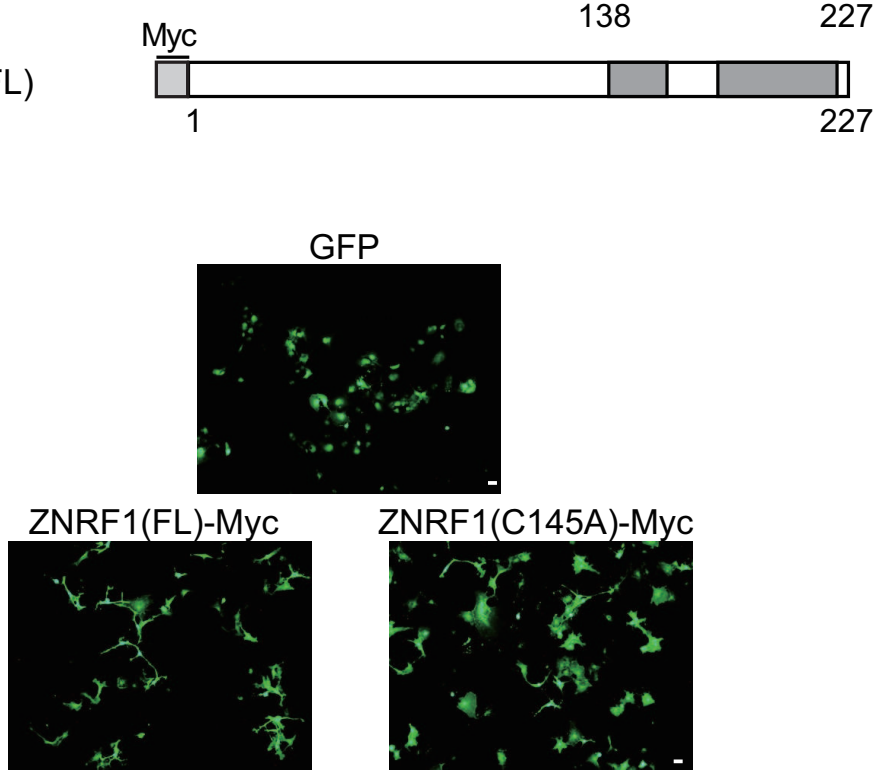

ZNRF1( $\Delta$ Zinc/RING)-Myc Myc-ZNRF1(138-227)
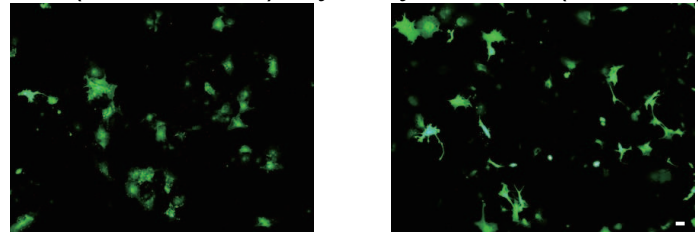

ZNRF1(C184A)-Myc
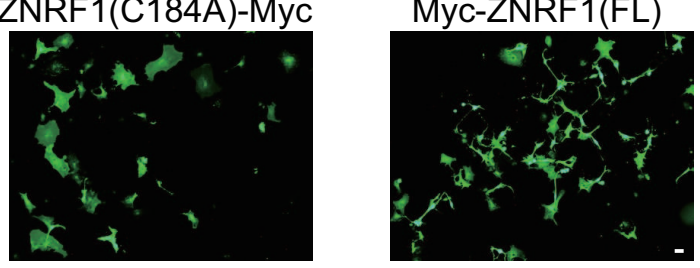

D
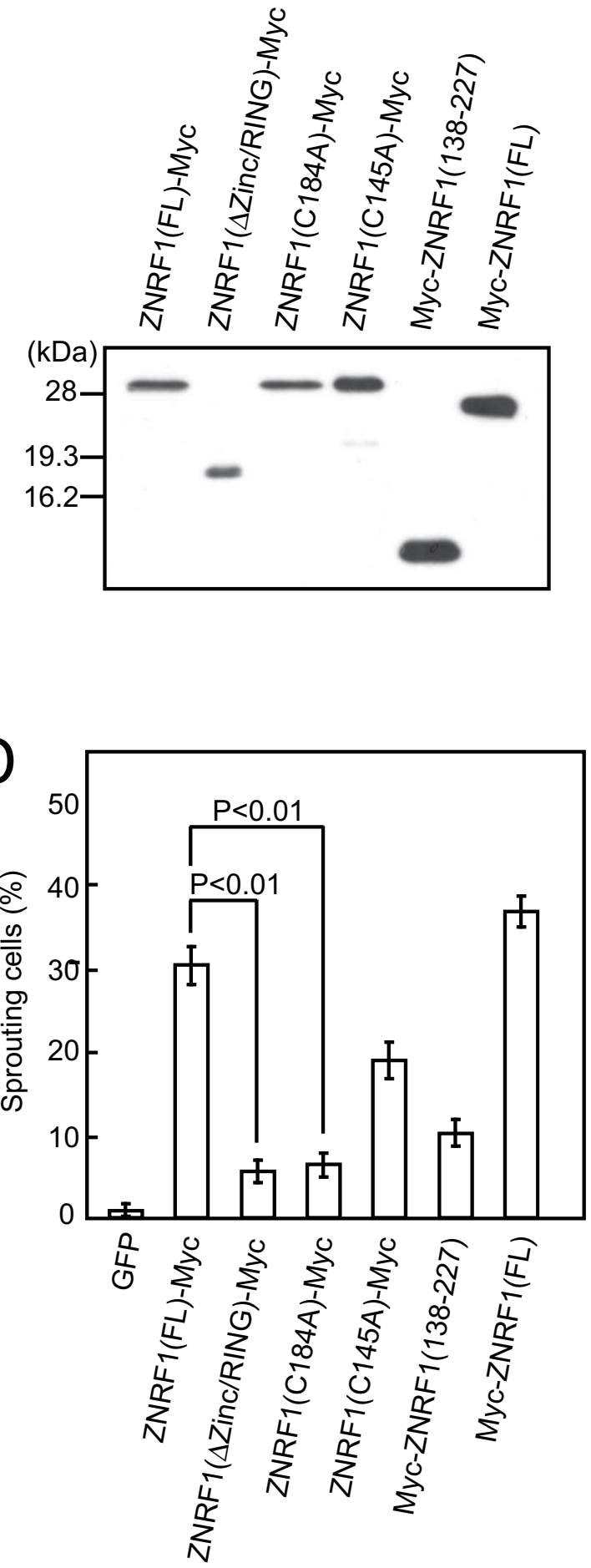

Yoshida et al. Fig. 2 
Aigure 4

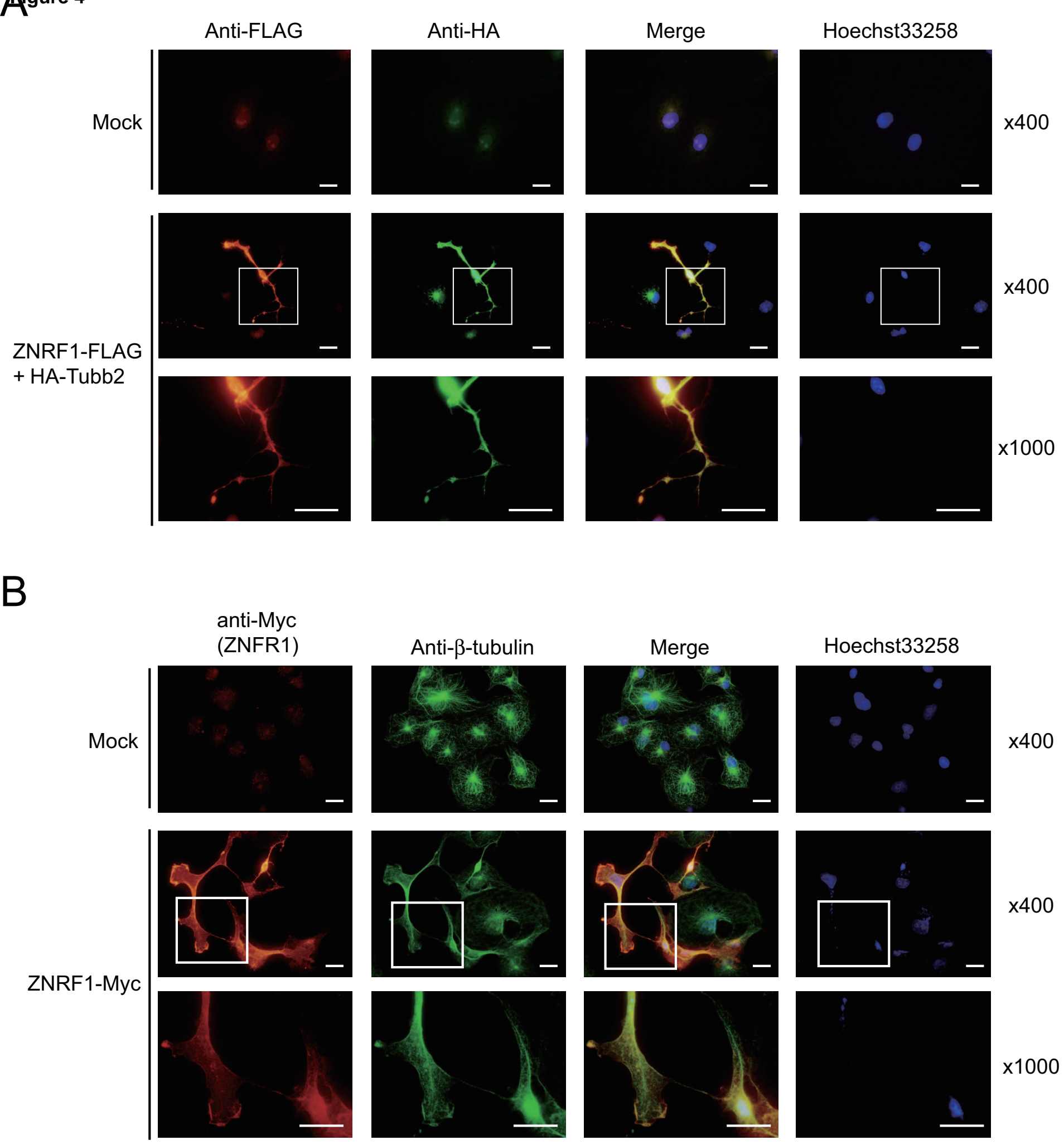

Yoshida et al. Fig. 4

4

ZNRF1-FLAG

(ZNFR1)
Hoechst33258

$x 400$

$x 1000$

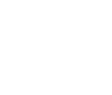

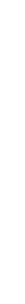

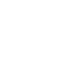

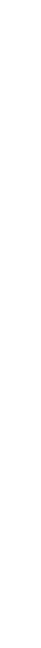

